\title{
Some aspects of probabilistic modeling, identification and propagation of uncertainties in computational mechanics
}

\author{
Christian Soize \\ Université Paris-Est, Laboratoire Modélisation et Simulation Multi-Echelle, \\ MSME UMR 8208 CNRS, \\ 5 bd Descartes, 77454 Marne-la-Vallée, Cedex 2, France, \\ christian.soize@univ-paris-est.fr
}

\begin{abstract}
In this paper, we present some aspects relative to the types of uncertainties, the variability of real systems, the types of probabilistic approaches and of the representations for the probabilistic models of uncertainties, the construction of the probabilistic models using the maximum entropy principle. We then present the nonparametric probabilistic approach of uncertainties for elliptic problems, for $3 D$ continuous dynamical systems with geometrical nonlinearities induced by large displacements and for low-and medium-frequency vibroacoustics of a complex system with experimental validations. Finally, a generalized probabilistic approach of uncertainties in computational dynamics using the random matrix theory and polynomial chaos decompositions is presented.

RÉSUMÉ. Dans cet article, on introduit les types d'incertitudes, la variabilité des systèmes réels, les types d'approches probabilistes, les types de représentation des modèles probabilistes des incertitudes, la construction des modèles probabilistes en utilisant le principe du maximum d'entropie. On présente ensuite l'approche probabiliste non paramétrique des incertitudes pour les problèmes elliptiques, pour l'élastodynamique 3D avec non-linéarités géométriques induites par les grands déplacements et la vibroacoustique basse et moyenne fréquence d'un système complexe avec validation expérimentale. Finalement, on présente une approche probabiliste généralisée des incertitudes pour en dynamique numérique en utilisant la théorie des matrices aléatoires et les décompositions sur les chaos polynômiaux.

KEYWORDS: Uncertainties, probabilistic modeling, uncertainties quantification, propagation of uncertainties, inverse problems, identification

MOTS-CLÉS : Incertitudes, modélisation probabiliste, quantification des incertitudes, propagation des incertitudes, problèmes inverses, identification
\end{abstract}

EJCM - 19/2010. Giens 2009, pages 25 to 40 


\section{Introduction concerning the probabilistic modeling of uncertainties}

\subsection{Uncertainties and variability}

The designed system is used to manufacture the real system and to construct the nominal computational model (also called the mean model) using a mathematicalmechanical modeling process for which the main objective is the prediction of the responses of the real system in its environment. The real system, submitted to a given environment, can exhibit a variability in its responses due to fluctuations in the manufacturing process and due to small variations of the configuration around a nominal configuration associated with the designed system. The mean model which results from a mathematical-mechanical modeling process of the design system, has parameters which can be uncertain. In this case, there are model-parameter uncertainties. In an other hand, the modeling process induces some modeling errors defined as the model uncertainties. It is important to take into account both the model-parameter uncertainties and model uncertainties to improve the predictions of computational models in order to use such a computational model to carry out robust optimization, robust design and robust updating with respect to uncertainties. Today, it is well understood that, as soon as the probability theory can be used, then the probabilistic approach of uncertainties is certainly the most powerful, efficient and effective tool for modeling and for solving direct and inverse uncertain problem. The developments presented in this paper are limited to the probabilistic approaches.

\subsection{Types of approach for probabilistic modeling of uncertainties}

The parametric probabilistic approach consists in modeling the uncertain parameters of the mean model by random variables and then in constructing the probabilistic model of these random variables using the available information. Such an approach is very well adapted and very efficient to take into account model-parameter uncertainties as soon as the probability theory can be used. Many works have been published in this field and a state-of-the-art can be found for instance in (Deodatis et al., 2008; Mace et al., 2005; Schueller, 2005a; Schueller, 2005b)). Nevertheless, the parametric probabilistic approach does not allow model uncertainties to be taken into account (see for instance (Beck et al., 1998)). A possible way to solve this difficult problem is the nonparametric probabilistic approach which has been introduced by (Soize, 2000; Soize, 2001) and for which extensions and developments have been presented in (Soize, 2003; Soize, 2005). This approach consists in replacing the matrices of the finite approximations of the operators of the boundary value problem by random matrices for which the mean values are equal to the corresponding matrices of the mean model and for which the probability distributions are constructed using only the available information. The nonparametric probabilistic approach then uses the random matrix theory (see for instance (Mehta, 1991)) and has required to introduce new ensembles of random matrices adapted to computational mechanics modeling (Soize, 2000; Soize, 2001; Soize, 2005; Mignolet et al., 2008a) and which 
have been constructed using the maximum entropy principle under the constraints defined by the available information (see Section 1.4). This approach has been applied and validated for direct problems of predictions, stochastic inverse problems of identification and robust optimization problems (Arnst et al., 2006; Capiez-Lernout et al., 2006; Chen et al., 2006; Cottereau et al., 2007; Capiez-Lernout et al., 2008; Desceliers et al., 2008; Durand et al., 2008; Pellissetti et al., 2008; Soize et al., 2008; Batou et al., 2009) for many situations, often with experimental validations. Extensions have been proposed, particularly in dynamic substructuring and in nonlinear dynamics (Chebli et al., 2004; Capiez-Lernout et al., 2005; Desceliers et al., 2004; Duchereau et al., 2006; Sampaio et al., 2007; Mignolet et al., 2008b; Batou et al., 2009). Very recently, a generalized probabilistic approach of uncertainties in computational dynamics using random matrices and polynomial chaos decompositions has been proposed (Soize, 2010).

\subsection{Types of representation for probabilistic modeling of uncertainties}

A fundamental question is the construction of prior probability models of uncertainties. Such a prior probability model can then be used to study the propagation of uncertainties through the mechanical system which is analyzed. If experimental measures are available for the mechanical system, then these measures can be used (1) to identify the parameters of the prior probability model (see for instance (Soize et al., 2008)) using, for instance, the maximum likelihood method (Serfling, 1980; Spall, 2003) or (2) to construct a posterior probability model (see for instance (Beck et al., 1998)) using, for instance, the Bayesian methods (see for instance (Bernardo et al., 2000; Kaipio et al., 2005; Spall, 2003; Congdon, 2007; Carlin et al., 2009)). Two methods can be used to construct the prior probability model of a random vector $\boldsymbol{A}$ belonging to the space $\mathcal{L}_{N}^{2}$ of all the second-order random variables defined on a probability space and with values in $\mathbb{R}^{N}$ (this random vector can result from a finite dimension approximation of a stochastic process or of a random field).

(i)- The first method is a direct approach which consists in directly constructing the probability distribution $P_{\boldsymbol{A}}(d \boldsymbol{a})$ on $\mathbb{R}^{N}$ in using, for instance, the maximum entropy principle (see Section 1.4).

(ii)- The second method is an indirect approach which consists in introducing a representation $\boldsymbol{A}=\boldsymbol{g}(\boldsymbol{X})$ for which $\boldsymbol{A} \in \mathcal{L}_{N}^{2}$ is the transformation by a deterministic nonlinear mapping $\boldsymbol{g}$ (which has to be constructed) of a $\mathbb{R}^{\nu}$-valued random variable $\boldsymbol{X}=\left(X_{1}, \ldots, X_{\nu}\right)$ whose probability distribution $P_{\boldsymbol{X}}(d \boldsymbol{x})$ is given and then is known. Then $P_{\boldsymbol{A}}$ is the transformation of $P_{\boldsymbol{X}}$ by the mapping $\boldsymbol{g}$. Two main types of methods can be used.

(ii.1)- The first one corresponds to the spectral methods such as the Polynomial Chaos representations (Wiener, 1938; Ghanem et al., 1991; Ghanem et al., 1996; LeMaitre et al., 2004; Soize et al., 2004; Nouy, 2007; Das et al., 2008; Arnst et al., 2008; Nouy et al., 2009; Soize et al., 2009) which can also be applied in infi- 
nite dimension for stochastic processes and random fields, which allow the effective construction of mapping $\boldsymbol{g}$ to be carried out and which allow any random variable $\boldsymbol{A}$ in $\mathcal{L}_{N}^{2}$, to be written as

$$
\boldsymbol{A}=\Sigma_{j_{1}=0}^{+\infty} \ldots \Sigma_{j_{\nu}=0}^{+\infty} \boldsymbol{a}^{j_{1}, \ldots, j_{\nu}} \psi_{j_{1}}\left(X_{1}\right) \times \ldots \times \psi_{j_{\nu}}\left(X_{\nu}\right)
$$

in which $\psi_{j}$ are given real polynomials and where $\boldsymbol{a}^{j_{1}, \ldots, j_{\nu}}$ are vectors in $\mathbb{R}^{N}$ which completely define mapping $\boldsymbol{g}$. The construction of $\boldsymbol{g}$ then consists in identifying the vector-valued coefficients $\boldsymbol{a}^{j_{1}, \ldots, j_{\nu}}$. If $\boldsymbol{X}$ is Gaussian, then the polynomials are the normalized Hermite polynomials. Today, many applications of such an approach have been carried out for direct and inverse problems, see for instance (Ghanem et al., 2005; Berveiller et al., 2006; Desceliers et al., 2006; Faverjon et al., 2006; Desceliers et al., 2007; LeMaitre et al., 2007; Arnst et al., 2008; Guilleminot et al., 2008)).

(ii.2)- The second one consists in introducing a prior algebraic representation $\boldsymbol{A}=$ $\widetilde{\boldsymbol{g}}(\boldsymbol{X}, \boldsymbol{w})$ in which $\boldsymbol{w}$ is a parameter with small dimension which has to be identified, where $\boldsymbol{X}$ id a vector-valued random variable with probability distribution $P_{\boldsymbol{X}}$, where $\widetilde{\boldsymbol{g}}$ is a given nonlinear mapping and where the vector-valued parameter $\boldsymbol{w}$ must be identified. For instance, tensor-valued random fields representations constructed with such an approach can be found in (Soize, 2006; Soize, 2008b).

Method (ii.1) allows any random vector $\boldsymbol{A}$ in $\mathcal{L}_{N}^{2}$ to be represented but the representation can require a very large number of coefficients yielding very difficult problems for their identification. In general, method (ii.2) does not allow any random vector $\boldsymbol{A}$ in $\mathcal{L}_{N}^{2}$ to be represented but allows a representation to be constructed in a subspace of $\mathcal{L}_{N}^{2}$ when $\boldsymbol{w}$ runs through all the admissible space (but, in opposite, the identification of $\boldsymbol{w}$ is realistic and efficient.

\subsection{Construction of the probabilistic models using the maximum entropy principle}

The measure of uncertainties using the entropy were introduced by (Shannon, 1948) in the framework of Information Theory. The use of the maximum entropy principle (that is to say the maximization of the level of uncertainties) to construct a prior probability model of a random variable under the constraints defined by the available information, were formalized by (Jaynes, 1957). This principle appears as a major tool to construct the prior probability model (1) of uncertain parameters of the mean model for the parametric probabilistic approach, (2) of both model-parameter uncertainties and modeling errors for the nonparametric probabilistic approach and (3) of the generalized approach of uncertainties corresponding to a full hybridization of the parametric and nonparametric probabilistic approaches.

Let $\boldsymbol{a}=\left(a_{1}, \ldots, a_{N}\right)$ be a real vector and let $\boldsymbol{A}=\left(A_{1}, \ldots, A_{N}\right)$ be a secondorder random variable with values in $\mathbb{R}^{N}$ whose probability distribution $P_{\boldsymbol{A}}$ is defined by a probability density function $\boldsymbol{a} \mapsto p_{\boldsymbol{A}}(\boldsymbol{a})$ on $\mathbb{R}^{N}$ with respect to $d \boldsymbol{a}=d a_{1} \ldots d a_{N}$ and which verifies the normalization condition $\int_{\mathbb{R}^{N}} p_{\boldsymbol{A}}(\boldsymbol{a}) d \boldsymbol{a}=1$. In fact, it is assumed that $\boldsymbol{A}$ is with values in any bounded or unbounded part $\mathcal{A}$ of $\mathbb{R}^{N}$ and conse- 
quently, the support of $p_{A}$ is $\mathcal{A}$. The available information defines a constraints equation on $\mathbb{R}^{\mu}$ written as $E\{\boldsymbol{g}(\boldsymbol{A})\}=\boldsymbol{f}$ in which $E$ is the mathematical expectation, $\boldsymbol{f}=\left(f_{1}, \ldots, f_{\mu}\right)$ is a given vector in $\mathbb{R}^{\mu}$ and where $\boldsymbol{a} \mapsto \boldsymbol{g}(\boldsymbol{a})=\left(g_{1}(\boldsymbol{a}), \ldots, g_{\mu}(\boldsymbol{a})\right)$ is a given function from $\mathbb{R}^{N}$ into $\mathbb{R}^{\mu}$. Let $\mathcal{C}$ be the set of all the probability density functions $\boldsymbol{a} \mapsto p_{\boldsymbol{A}}(\boldsymbol{a})$ defined on $\mathbb{R}^{N}$ with values in $\mathbb{R}^{+}$verifying the normalization condition and the constraints equation $E\{\boldsymbol{g}(\boldsymbol{A})\}=\boldsymbol{f}$. The maximum entropy principle consists in finding $p_{\boldsymbol{A}}$ in $\mathcal{C}$ which maximizes the entropy (that is to say the uncertainties),

$$
p_{\boldsymbol{A}}=\arg \max _{p \in \mathcal{C}} S(p) \quad, \quad S(p)=-\int_{\mathbb{R}^{N}} p(\boldsymbol{a}) \log (p(\boldsymbol{a})) d \boldsymbol{a}
$$

in which $S(p)$ is the entropy of the probability density function $p$. Introducing the Lagrange multiplier $\lambda \in \mathcal{L}_{\mu} \subset \mathbb{R}^{\mu}$ associated with the constraints where $\mathcal{L}_{\mu}$ is the subset of $\mathbb{R}^{\mu}$ of all the admissible values for $\lambda$, it can easily be seen that the solution of the optimization problem can be written as

$$
p_{\boldsymbol{A}}(\boldsymbol{a})=c_{0} \mathbf{1}_{\mathcal{A}}(\boldsymbol{a}) \exp (-<\boldsymbol{\lambda}, \boldsymbol{g}(\boldsymbol{a})>) \quad, \quad \forall \boldsymbol{a} \in \mathbb{R}^{N}
$$

in which $\langle\boldsymbol{x}, \boldsymbol{y}\rangle=x_{1} y_{1}+\ldots+x_{\mu} y_{\mu}$ and where $\mathbf{1}_{\mathcal{A}}$ is the indicatrix function of set $\mathcal{A}$. The normalization constant $c_{0}$ and the Lagrange multiplier $\boldsymbol{\lambda}$ are calculated in solving a nonlinear vectorial algebraic equation deduced from the normalization condition and from the constraints equation. This algebraic equation can be solved using appropriated algorithms. Then, it is necessary to construct a generator of independent realizations of random variable $\boldsymbol{A}$ whose probability density function is that which has been built. In small dimension ( $\mathrm{N}$ is a few units), there is no difficulty. In high dimension ( $\mathrm{N}$ hundreds or thousands), there are two major difficulties. The first one is related to the calculation of an integral in high dimension of the type $c_{0} \int_{\mathcal{A}} \boldsymbol{g}(\boldsymbol{a}) \exp (-<\boldsymbol{\lambda}, \boldsymbol{g}(\boldsymbol{a})>) d \boldsymbol{a}$ which is necessary to implement the algorithm for computing $c_{0}$ and $\lambda$. The second one is the construction of the generator once $c_{0}$ et $\boldsymbol{\lambda}$ have been calculated. These two aspects can be solved using the Markov Chain Monte Carlo methods (MCMC) (see for instance (Kaipio et al., 2005; MacKeown, 1997; Spall, 2003)). The transition kernel of the homogeneous (stationary) Markov chain of the MCMC method can be constructed using the Metropolis-Hastings algorithm (Hastings, 1970) or the Gibbs algorithm (Geman et al., 1984) which is a slightly different algorithm for which the kernel is directly derived from the transition probability density function and for which the Gibbs realizations are always accepted. These two algorithms construct the transition kernel for which the invariant measure is $P_{A}$. In general, these algorithms are effective but can not be when there are regions of attraction that do not correspond to the invariant measure. These situations can not be easily detected and are time consuming. Recently, a new approach (Soize, 2008a) of the class of the Gibbs method has been proposed to avoid these difficulties and is based on the introduction of an Itô stochastic differential equation whose unique invariant measure is $P_{\boldsymbol{A}}$ and is the explicit solution of a Fokker-Planck equation (Soize, 1994). The algorithm is then obtained by discretization of the Itô equation. 


\section{Nonparametric probabilistic approach of uncertainties for elliptic boundary value problems}

For the general case of elliptic boundary value problems, the nonparametric probabilistic approach of uncertainties can fe found in (Soize, 2009). The mean computational model resulting from the finite element discretization of the strictly elliptic boundary value problem under consideration is written as $[\underline{\mathbb{K}}][\underline{x}]=[b]$ in which $[\underline{\mathbb{K}}]$ is a positive-definite $(n \times n)$ sparse real matrix, where $[\underline{x}]$ is a $(n \times m)$ real matrix made up of the unknowns and where [b] is a given $(n \times m)$ real matrix. The nonparametric probabilistic approach cannot directly be used, one hand because of the presence of topological zeros in the sparse matrix $[\underline{\mathbb{K}}]$ and partly because for problems in high dimension the large full random matrix $[\mathbb{K}]$ could not be managed (if $n=10^{7}$, then there is $0.5 \times 10^{14}$ nonzero elements!). It is therefore necessary to build a reduced model of dimension $N \ll n$ knowing that a reduction of modal type would not be effective with respect to the convergence in $N$. The proposed approach in (Soize, 2009) consists in associating with the mean computational model, the stochastic equation $[\mathbb{K}][\boldsymbol{X}]=[b]$ and a family $\left\{\left[\mathbb{K}^{N}\right], N=1, \ldots, n\right\}$ of $(n \times n)$ random matrices belonging to the set $\mathrm{SE}^{+}$ defined in (Soize, 2005) such that the random solution $\left[\boldsymbol{X}^{N}\right]$ of the stochastic equation $\left[\mathbb{K}^{N}\right]\left[\boldsymbol{X}^{N}\right]=[b]$ is a second-order solution $E\left\{\left\|\left[\boldsymbol{X}^{N}\right]\right\|_{F}^{2}\right\}=c<+\infty$ such that $\lim _{N \rightarrow n} E\left\{\left\|\left[\boldsymbol{X}^{N}\right]-[\boldsymbol{X}]\right\|_{F}^{2}\right\}=0$, where $N \leq n$ is the dimension of the reduced-order model. For fixed $N$, random matrix $\left\{\left[\mathbb{K}^{N}\right]\right.$ is defined by $\left[\mathbb{K}^{N}\right]=\left\{\left[\Phi^{N}\right]\left(\left[\boldsymbol{K}_{N}\right]^{-1}-\left[\underline{K}_{N}\right]^{-1}\right)\left[\Phi^{N}\right]^{T}+[\underline{\mathbb{K}}]^{-1}\right\}^{-1}$ with $\left[\Phi^{N}\right]$ the $(n \times N)$ real matrix whose columns are the $N$ orthonormal eigenvectors of $[\underline{\mathbb{K}}]$ associated with the $N$ first positive eigenvalues $0<\lambda_{1} \leq \lambda_{2} \leq \ldots \leq \lambda_{N}$. The diagonal matrix $\left[\underline{K}_{N}\right]$ made up of the eigenvalues $\lambda_{1}, \ldots \lambda_{N}$ are written as $\left[\underline{K}_{N}\right]=\left[\underline{L}_{N}\right]^{T}\left[\underline{L}_{N}\right]$ and the full random matrix $\left[\boldsymbol{K}_{N}\right]$ is such that $\left[\boldsymbol{K}_{N}\right]=\left[\underline{L}_{N}\right]^{T}\left[\boldsymbol{G}_{N}\right]\left[\underline{L}_{N}\right]$ in which $\left[\boldsymbol{G}_{N}\right]$ is the $(N \times N)$ full random matrix belonging to the set $\mathrm{SG}^{+}$defined in (Soize, 2005). We then have $E\left\{\left[\boldsymbol{K}_{N}\right]\right\}=\left[\underline{K}_{N}\right]$. Obviously, the $(n \times n)$ full random matrix $\left[\boldsymbol{K}_{N}\right]$ is never assembled and the specialized algorithm detailed in (Soize, 2009) is used.

As an example, we consider the following problem in linear elastostatics. The finite element mesh of the domain is shown in Figure 1 (a) and there are 17, 355 DOF. The material is linear elastic homogeneous and isotropic. There are Dirichlet conditions on part of the boundary and there are $m=4$ independent static loads applied on the outer lateral surface of the cylinder. The level of uncertainty is defined by the value of the dispersion parameter $\delta$ of the random matrix $\left[\boldsymbol{G}_{N}\right]$. The stochastic solver is based on the use of the Monte Carlo method for which $n_{s}=20,000$ independent realizations are used. Figure 1 (b) shows the mean square convergence of the random solution as a function of dimension $N$ of the reduced-order model and for $\delta=0.5$. Convergence is reached for $N \geq 300$. Let $U$ (or $V$ ) be the random variable representing the component of the random response for which the mean value (or the standard deviation) is the largest. Let $F_{U}(u)=\operatorname{Proba}\{U \leq u\}$ (or $F_{V}(v)$ ) be the cumulative distribution function of $U$ (or $V$ ). For $n_{s}=20,000$ and $N=500$, Figures 2 (a) and 
(b) show the graphs of functions $u \mapsto \log _{10} F_{U}(u)$ and $v \mapsto \log _{10} F_{V}(v)$ for several values of $\delta$.

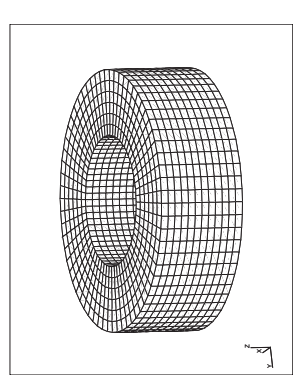

(a)

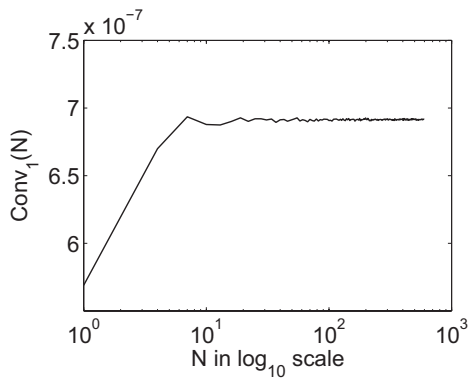

(b)

Figure 1. Finite element mesh (a). Mean-square convergence with respect to $N(b)$

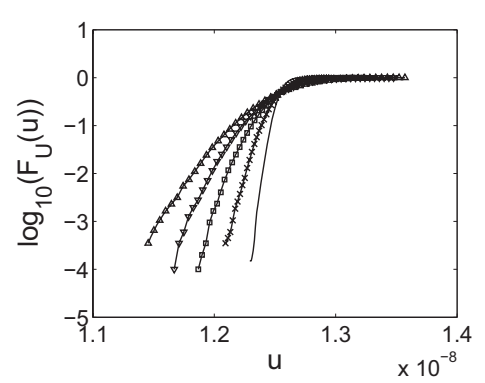

(a)

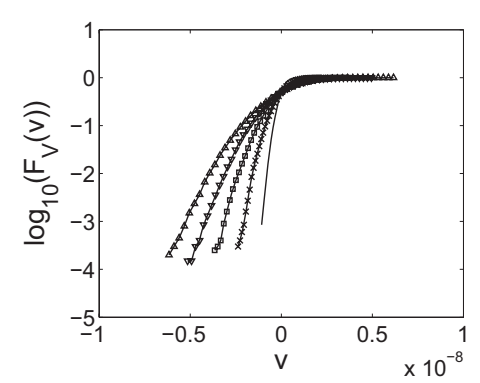

(b)

Figure 2. Graph of $u \mapsto \log _{10} F_{U}(u)$ (a) and graph of $v \mapsto \log _{10} F_{V}(v)$ (b) for $\delta=0.1$ (no symbol), $\delta=0.2(x), \delta=0.3$ (square), $\delta=0.4$ (down triangle), $\delta=0.5$ (up triangle)

\section{Nonparametric probabilistic approach of uncertainties in 3D elastodynamics with geometric nonlinearities}

The nonparametric probabilistic approach of uncertainties for the general case of $3 \mathrm{D}$ elastodynamics with large displacements and with linear material behavior, that is to say, in the presence of geometric nonlinearities has been introduced in (Mignolet et al., 2008b). The nonparametric probabilistic approach of uncertainties for this nonlinear dynamical system yields the following stochastic reduced-order model for the displacement random field $\boldsymbol{U}(x, y, z, t)=\sum_{i=1}^{n} Q_{i}(t) \underline{\varphi}_{i}(x, y, z)$ and the stresses random field $\boldsymbol{S}(t)=\underline{\boldsymbol{s}}^{0}+\sum_{i=1}^{n} \underline{\boldsymbol{s}}_{i}^{(1)} Q_{i}(t)+\sum_{i, j=1}^{n}\left[\underline{S}^{(2)}\right]_{i j} Q_{i}(t) Q_{j}(t)$ in which 
the random vector $\boldsymbol{Q}(t)$ of the generalized coordinates verifies the nonlinear stochastic differential equation $\left[\boldsymbol{M}_{n}\right] \ddot{\boldsymbol{Q}}(t)+\left[\boldsymbol{D}_{n}\right] \dot{\boldsymbol{Q}}(t)+\boldsymbol{K}_{N L}(\boldsymbol{Q}(t))=\boldsymbol{F}(t)$, in which the vector of the generalized nonlinear elastic forces is written as $\left\{\boldsymbol{K}_{N L}(\boldsymbol{q})\right\}_{i}=$ $\sum_{j=1}^{n}\left[\boldsymbol{K}^{(1)}\right]_{i j} q_{j}+\sum_{j, \ell=1}^{n} \boldsymbol{K}_{i j \ell}^{(2)} q_{j} q_{\ell}+\sum_{j, \ell, p=1}^{n} \boldsymbol{K}_{i j \ell p}^{(3)} q_{j} q_{\ell} q_{p}$, and where the random matrix $\left[\boldsymbol{K}_{B}\right]$ is written as $\left[\boldsymbol{K}_{B}\right]=\left[\begin{array}{ll}{\left[\boldsymbol{K}^{(1)}\right]} & {\left[\widetilde{\boldsymbol{K}}^{(2)}\right]} \\ {\left[\widetilde{\boldsymbol{K}}^{(2)}\right]^{T}} & 2\left[\widetilde{\boldsymbol{K}}^{(3)}\right]\end{array}\right]$. The random matrices $\left[\boldsymbol{M}_{n}\right],\left[\boldsymbol{D}_{n}\right]$ and $\left[\boldsymbol{K}_{B}\right]$, for which the level of uncertainties is controlled by $\delta_{M}, \delta_{D}$ and $\delta_{K_{B}}$, are statistically independent, symmetric and positive definite almost surely and belong to ensemble $\mathrm{SE}^{+}$. The random matrices $\left[\boldsymbol{K}^{(1)}\right],\left[\widetilde{\boldsymbol{K}}^{(2)}\right]$ and $\left[\widetilde{\boldsymbol{K}}^{(3)}\right]$ are statistically dependent and are deduced from random matrix $\left[\boldsymbol{K}_{B}\right]$. The random tensors $\boldsymbol{K}_{i j \ell}^{(2)}$ and $\boldsymbol{K}_{i j \ell p}^{(3)}$ are statistically dependent and are deduced from $\left[\widetilde{\boldsymbol{K}}^{(2)}\right]$ and $\left[\widetilde{\boldsymbol{K}}^{(3)}\right]$. The construction of the random coefficients of the nonlinear terms in the stochastic reduced model requires to know the corresponding deterministic coefficients of the reduced mean model. These coefficients can be calculated using the method developed in (Muravyov et al., 2003; Mignolet et al., 2008b) for general nonlinear dynamical systems in large displacements and only requires the use of any commercial software allowing nonlinear elastic analyses to be performed.

As an example, we consider a steel straight beam, fixed at both ends, with length $0.2286 \mathrm{~m}, 0.0127 \mathrm{~m}$ width and $0.000775 \mathrm{~m}$ thickness. The excitation is a point force applied to the center of the beam and has flat spectrum over the band [ $-2000,2000] \mathrm{Hz}$. The beam is discretized in 40 CBEAM finite elements with Nastran. The elastic modes of the linearized system (12 plane modes and 10 transverse modes) are chosen so that the modal response converged. The values of the parameters $\delta_{M}=\delta_{D}=0$ and $\delta_{K_{B}}$ are chosen for that the first natural frequency (transverse mode) is $4 \%$ of the coefficient of variation. Figure 3 shows the results for the random spectrum of the random transverse response in the middle of the beam.

\section{Nonparametric probabilistic approach in low- and medium-frequency vibroacoustics and experimental validation}

The nonparametric probabilistic approach of uncertainties for complex vibroacoustic systems with an application to an automotive vehicle can be found in (Durand et al., 2008). There are uncertainties in the vibroacoustic computational model for the structure, for the internal acoustic cavity and for the vibroacoustic coupling interface. The level of uncertainties are identified using experiments. A unique vibroacoustic computational model is developed for one type of car with several optional extra that induces variability in the vibroacoustic responses of the real system. The goal is the prediction of sound pressure in the acoustic cavity induced by the rotation of the engine for regimes $[1500,4800] \mathrm{rpm}$ corresponding to the frequency band $[50,160] \mathrm{Hz}$. The structure-borne excitations are dynamic forces applied to the engine mounts. The vibroacoustic system consists of a linear viscoelastic structure coupled with an acoustic cavity filled with a dissipative acoustic fluid. In the frequency domain $\omega$, the dis- 


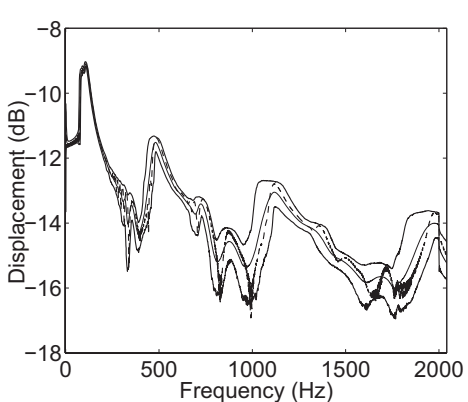

(a)

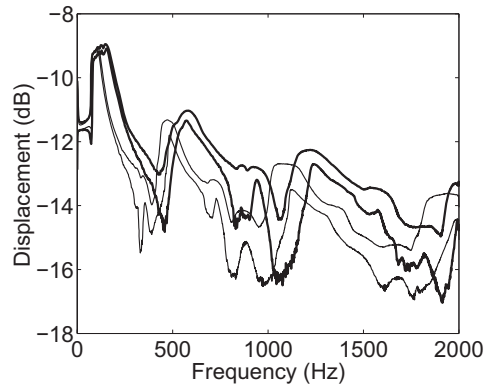

(b)

Figure 3. (a): mean model (very thin dashed line), statistical mean value (very thin solid line), confidence region at 95\% (upper and lower thin solid lines). (b): confidence region at $95 \%$ for two response levels; low amplitude as figure (a) (upper and lower thin solid lines); high amplitude equals to $2.25 \times$ the low amplitude case (upper and lower thick solid lines)

placement vector of the structure is $\underline{\boldsymbol{u}}(\omega)$ with $n_{s}$ DOF. The acoustic-pressure vector is $\boldsymbol{p}(\omega)$ with $n_{f}$ DOF. The reduced mean model is constructed using the $N_{s}$ first elastic modes $\underline{\varphi}_{\alpha}$ of the structure in vacuo and the $N_{f}$ first acoustic modes $\underline{\psi}_{\beta}$ of the acoustic cavity with rigid walls. The reduced mean model is then written as (Ohayon et al., 1998), $\underline{\boldsymbol{u}}(\omega)=\sum_{\alpha=1}^{N_{s}} \underline{q}_{\alpha}^{s}(\omega) \underline{\varphi}_{\alpha}$ and $\underline{\boldsymbol{p}}(\omega)=\sum_{\beta=1}^{N_{f}} \underline{q}_{\beta}^{f}(\omega) \underline{\boldsymbol{\psi}}_{\beta}$ with

$$
\left[\begin{array}{cc}
{\left[\underline{A}_{s}(\omega)\right]} & {[\underline{C}]} \\
\omega^{2}[\underline{C}]^{T} & {\left[\underline{A}_{f}(\omega)\right]}
\end{array}\right]\left[\begin{array}{l}
\underline{\boldsymbol{q}}^{s}(\omega) \\
\underline{\boldsymbol{q}}^{f}(\omega)
\end{array}\right]=\left[\begin{array}{l}
\underline{\boldsymbol{f}}^{s}(\omega) \\
\underline{\boldsymbol{f}}^{f}(\omega)
\end{array}\right],
$$

where $\left[\underline{A}_{s}(\omega)\right]=-\omega^{2}\left[\underline{M}_{s}\right]+i \omega\left[\underline{D}_{s}(\omega)\right]+\left[\underline{K}_{s}(\omega)\right]$ is the generalized dynamic stiffness of the structure, $\left[\underline{A}_{f}(\omega)\right]=-\omega^{2}\left[\underline{M}_{f}\right]+i \omega\left[\underline{D}_{f}\right]+\left[\underline{K}_{f}\right]$ is the generalized acoustic stiffness of the acoustic cavity and the $\left(N_{s} \times N_{f}\right)$ rectangular matrix $[\underline{C}]$ is the generalized vibroacoustic coupling matrix. The use of the nonparametric probabilistic approach of uncertainties yields the following stochastic reduced model. For all $\omega$ fixed in the frequency band of analysis, the random response is represented by the random complex vectors $\boldsymbol{U}(\omega)=\sum_{\alpha=1}^{N_{s}} Q_{\alpha}^{s}(\omega) \underline{\varphi}_{\alpha}$ and $\boldsymbol{P}(\omega)=\sum_{\beta=1}^{N_{f}} Q_{\beta}^{f}(\omega) \underline{\boldsymbol{\psi}}_{\beta}$ such that

$$
\left[\begin{array}{cc}
{\left[\boldsymbol{A}_{s}(\omega)\right]} & {[\boldsymbol{C}]} \\
\omega^{2}[\boldsymbol{C}]^{T} & {\left[\boldsymbol{A}_{f}(\omega)\right]}
\end{array}\right]\left[\begin{array}{l}
\boldsymbol{Q}^{s}(\omega) \\
\boldsymbol{Q}^{f}(\omega)
\end{array}\right]=\left[\begin{array}{l}
\underline{\boldsymbol{f}}^{s}(\omega) \\
\underline{\boldsymbol{f}}^{f}(\omega)
\end{array}\right]
$$

with $\left[\boldsymbol{A}_{s}(\omega)\right]=-\omega^{2}\left[\boldsymbol{M}_{s}\right]+i \omega\left[\boldsymbol{D}_{s}(\omega)\right]+\left[\boldsymbol{K}_{s}(\omega)\right],\left[\boldsymbol{A}_{f}(\omega)\right]=-\omega^{2}\left[\boldsymbol{M}_{f}\right]+i \omega\left[\boldsymbol{D}_{f}\right]+$ $\left[\boldsymbol{K}_{f}\right]$ and $[\boldsymbol{C}]$ random matrices such that $\left[\boldsymbol{M}_{s}\right],\left[\boldsymbol{D}_{s}(\omega)\right],\left[\boldsymbol{K}_{s}(\omega)\right]$ belong to ensemble $\mathrm{SE}^{+}$, where $\left[\boldsymbol{M}_{f}\right]$ belongs to $\mathrm{SE}^{+}$but where $\left[\boldsymbol{D}_{f}\right]$ and $\left[\boldsymbol{K}_{f}\right]$ belong to $\mathrm{SE}^{+0}$, and 
finally, where $[\boldsymbol{C}]$ belongs to $\mathrm{SE}_{\mathrm{inv}}$, these three ensembles of full random matrices being defined in (Soize, 2005). The probability distribution is completely defined for each ensemble of random matrices and only depends on the dimension of the random matrix, on its mean value (for instance $E\left\{\left[\boldsymbol{M}_{s}\right]\right\}=\left[\underline{M}_{s}\right]$, etc) and on a dispersion parameter which controls the level of uncertainties. These dispersion parameters are denoted by $\delta_{M_{s}}, \delta_{D_{s}}, \delta_{K_{s}}, \delta_{M_{f}}, \delta_{D_{f}}, \delta_{K_{f}}$ and $\delta_{C}$. The stochastic solver is based on the use of the Monte Carlo method. The means-square convergence is studied with respect to the number of independent realizations and with respect to the reduced-order model dimensions $N_{s}$ and $N_{f}$. The mean computational model of the automotive vehicle is made up of a finite element model having 978,733 DOF in displacement for the structure (see Figure 4 (a) and of a finite element model having 8, 139 DOF in pressure for the acoustic cavity (see Figure 4 (b)), the mesh being compatible on the vibroacoustic coupling interface. The reduced mean computational model has 1,722 elastic

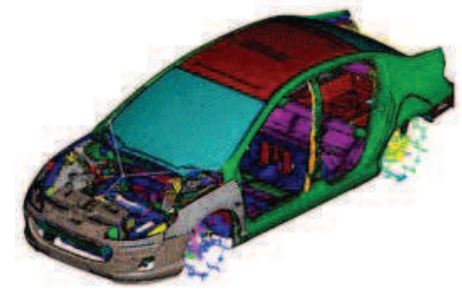

(a)

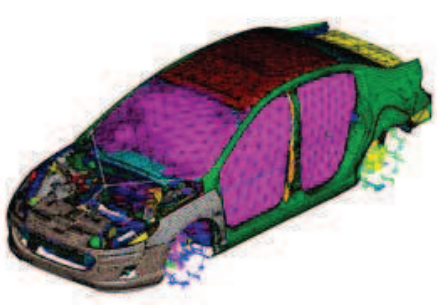

(b)

Figure 4. Finite element mesh for the structure (a) and for the acoustic cavity (b)

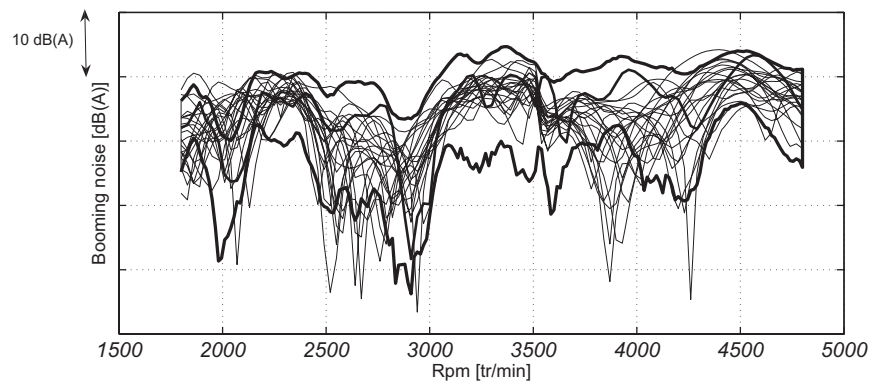

Figure 5. Acoustic-pressure modulus at a given point in the acoustic cavity for a given engine excitation as a function of the frequency (rotation per minute). Measurements (20 thin solid lines). Reduced mean model (mid thick solid line). Confidence region at 0.95 in $d B A$ (between upper and lower thick solid lines)

modes for the structure and 57 acoustic modes for the cavity. The level of uncertainties are represented by $\delta=\left\{\delta_{M_{s}}, \delta_{D_{s}}, \delta_{K_{s}}\right\}$ for the structure, by $\delta_{f}=\delta_{M_{f}}=\delta_{D_{f}}=\delta_{K_{f}}$ 
for the acoustic cavity and by $\delta_{C}$ for the vibroacoustic coupling. The dispersion parameters $\delta_{f}$ and $\delta$ have been identified from experimental measures performed on 30 cars for acoustics and 20 cars for vibrations (Durand et al., 2008). Figure 5 validates all of the stochastic modeling and displays the confidence region at 0.95 for the sound pressure at a given point in the cavity and for a given excitation of the engine. The calculations are done using the stochastic reduced vibroacoustic model for the experimentally identified dispersion parameters. The Monte Carlo method is used with $m=600$ independent realizations.

\section{Generalized probabilistic approach of uncertainties in computational dynamics using random matrices and polynomial chaos decompositions}

A generalized probabilistic approach of uncertainties has recently been proposed (Soize, 2010) for computational model in structural linear dynamics and can be extended without difficulty to computational linear vibroacoustics and to computational nonlinear structural dynamics. This method is an extension of the nonparametric probabilistic approach of uncertainties presented in the previous sections and allows the prior probability model of each type of uncertainties (model-parameter uncertainties and modeling errors) to be separately constructed and identified. The modeling errors are not taken into account with the usual output-prediction-error method (Beck et al., 1998) but with the nonparametric probabilistic approach of modeling errors introduced above and based on the use of the random matrix theory. A chaos decomposition (Ghanem et al., 1991) with random coefficients (Soize et al., 2009)is used to represent the prior probability model of random responses. The random germ is related to the prior probability model of model-parameter uncertainties. The random coefficients are related to the prior probability model of modeling errors and then depends on the random matrices introduced by the nonparametric probabilistic approach of modeling errors. If experimental data are available, then the prior probability model of the random coefficients can be improved in constructing a posterior probability model using the Bayesian methods (see for instance (Bernardo et al., 2000; Kaipio et al., 2005; Spall, 2003; Congdon, 2007; Carlin et al., 2009)). The theory, an identification procedure of each type of uncertainties and a numerical validation are presented in (Beck et al., 1998). We present an example of the generalized approach as an illustration of this generalized probabilistic approach of uncertainties. The designed system is a slender cylindrical elastic medium with length $10 \mathrm{~m}$ and has a rectangular section with height $1.1 \mathrm{~m}$ and width $1.6 \mathrm{~m}$. The elastic medium is made of a composite material. The displacement field is zero on the part of the two end sections. The frequency band of analysis is $B=] 0,1200] \mathrm{Hz}$. A point load is applied close to the middle of the slender cylinder with a flat spectrum on $B$. We are interested in the transversal displacement of the neutral line at an observation point belonging to the neutral line and close to the end section. A reference solution of the real system has been constructed in developing a 3D elastic model of the real system. The mean model is made up of a damped homogeneous Euler elastic beam with length $10 \mathrm{~m}$ and simply supported. From the Euler beam theory, the first eigenvalue (square of the first eigenfrequency) 
can be written $\lambda_{1}=a_{1} \underline{x}$. The model-parameter uncertainties leads us to model $x$ by a random variable $X$. The prior probability model of random variable $X$ is constructed using the maximum entropy principle and yields a Gamma probability distribution for which the mean value is given and for which the dispersion is controlled by the coefficient of variation $\delta_{X}$. The identification of parameter $\delta_{X}$ is performed using the maximum likelihood method for the lowest random eigenvalue $\Lambda_{1}$ and yields $\delta_{X}=0.093$. We consider the stochastic reduced model constructed (1) with the parametric probabilistic approach of uncertain parameter $X$ for which $\delta_{X}=0.093$ and (2) with the nonparametric probabilistic approach of modeling errors for the mass and stiffness operators. Consequently, the dispersion parameters describing the statistical fluctuations induced by modeling errors are $\delta_{M}$ and $\delta_{K}$. The method used for the identification of $\delta_{M}$ and $\delta_{K}$ is explained in (Soize, 2010) and yields $\delta_{M}=0.9$ and $\delta_{K}=0.15$. Figure 6 displays the comparisons between the response of the mean model, the ref-

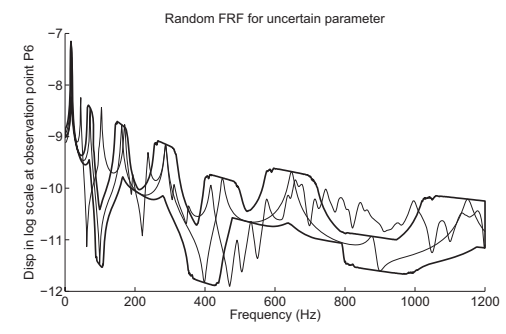

(a)

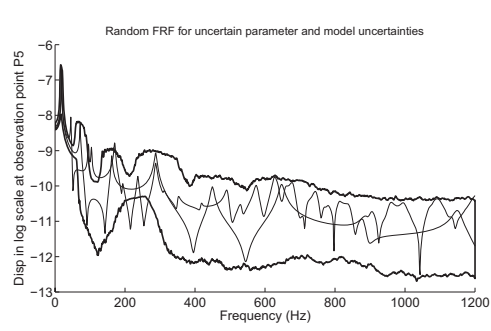

(b)

Figure 6. Confidence region of the response at 0.98 (between upper and lower thick solid lines), reference response (mid solid line), response of the mean model (thin solid line) at observation point. (a): parametric probabilistic approach. (b): generalized probabilistic approach

erence response of the real system and the confidence region of the random response (1) calculated with the parametric probabilistic approach with $\delta_{X}=0.093$ (figure (a)) and (2) calculated with the generalized probabilistic approach, that is to say, with the parametric probabilistic approach of model-parameter uncertainties for $\delta_{X}=0.093$ and with the nonparametric probabilistic approach of modeling errors for $\delta_{M}=0.9$ and $\delta_{K}=0.15$. These figures show that the coupling of the two probabilistic approaches for model-parameter uncertainties and modeling errors allow the quality of the prediction to be considerably improved. The method proposed allows the role played by each type of uncertainties to be separately quantified.

\section{Conclusions}

This paper has presented a very brief survey concerning advances in uncertainties modeling, their quantification and their propagation through linear and nonlinear mechanical systems in static and dynamics. 


\section{Acknowledgements}

The given illustrations concerning automotive vehicles and nonlinear elastodynamics come from recent papers carried out in collaboration and the author thanks the co-authors of these papers, Jean-François Durand, Laurent Gagliardini from PSA Peugeot-Citroen and Marc Mignolet from Arizona State University.

\section{References}

Arnst M., Clouteau D., Chebli H., Othman R., Degrande G., “ A nonparametric probabilistic model for ground-borne vibrations in buildings", Probabilistic Engineering Mechanics, vol. 21, n 1, p. 18-34, 2006.

Arnst M., Ghanem R., " Probabilistic equivalence and stochastic model reduction in multiscale analysis", Computer Methods in Applied Mechanics and Engineering, vol. 197, p. 35843592, 2008.

Batou A., Soize C., " Identification of stochastic loads applied to a non-linear dynamical system using an uncertain computational model and experimental responses", Computational Mechanics, vol. 43, n 4, p. 559-571, 2009.

Beck J. L., Katafygiotis L. S., “ Updating models and their uncertainties. I: Bayesian statistical framework”, Journal of Engineering Mechanics, vol. 124, n 4, p. 455-461, 1998.

Bernardo J., Smith A. F. M., Bayesian Theory, John Wiley \& Sons, Chichester, 2000.

Berveiller M., Sudret B., Lemaire M., " Stochastic finite element: a non-intrusive approach by regression”, European Journal of Computational Mechanics, vol. 15, p. 81-92, 2006.

Capiez-Lernout E., Pellissetti M., Pradlwarter H., Schueller G. I., Soize C., “ Data and model uncertainties in complex aerospace engineering systems", Journal of Sound and Vibration, vol. 295, n 3-5, p. 923-938, 2006.

Capiez-Lernout E., Soize C., “ Robust design optimization in computational mechanics”, Journal of Applied Mechanics - Transactions of the ASME, vol. 75, n², p. 021001-1 - 021001$11,2008$.

Capiez-Lernout E., Soize C., Lombard J.-P., Dupont C., Seinturier E., “ Blade manufacturing tolerances definition for a mistuned industrial bladed disk", Journal of Engineering for Gas Turbines and Power, vol. 127, n³ 3, p. 621-628, 2005.

Carlin B. P., Louis T. A., Bayesian Methods for Data Analysis, Third Edition, CRC Press, Boca Raton, 2009.

Chebli H., Soize C., " Experimental validation of a nonparametric probabilistic model of non homogeneous uncertainties for dynamical systems", Journal of the Acoustical Society of America, vol. 115, n 2, p. 697-705, 2004.

Chen C., Duhamel D., Soize C., “Probabilistic approach for model and data uncertainties and its experimental identification in structural dynamics: Case of composite sandwich panels", Journal of Sound and Vibration, vol. 294, n 1-2, p. 64-81, 2006.

Congdon P., Bayesian Statistical Modelling, Second Edition, John Wiley \& Sons, Chichester, 2007. 
Cottereau R., Clouteau D., Soize C., " Construction of a probabilistic model for impedance matrices", Computer Methods in Applied Mechanics and Engineering, vol. 196, n 17-20, p. 2252-2268, 2007.

Das S., Ghanem R., Spall J. C., “ Asymptotic sampling distribution for polynomial chaos representation from data: a maximum entropy and fisher information approach", SIAM Journal on Scientific Computing, vol. 30, n 5, p. 2207-2234, 2008.

Deodatis G., Spanos P. D., " 5th International Conference on Computational Stochastic Mechanics", Special issue of the Probabilistic Engineering Mechanics, vol. 23, n 2-3, p. $103-$ 346, 2008.

Desceliers C., Ghanem R., Soize C., “ Maximum likelihood estimation of stochastic chaos representations from experimental data", International Journal for Numerical Methods in Engineering, vol. 66, n 6, p. 978-1001, 2006.

Desceliers C., Soize C., Cambier S., " Non-parametric - parametric model for random uncertainties in nonlinear structural dynamics - Application to earthquake engineering", Earthquake Engineering and Structural Dynamics, vol. 33, n 3, p. 315-327, 2004.

Desceliers C., Soize C., Ghanem R., "Identification of chaos representations of elastic properties of random media using experimental vibration tests", Computational Mechanics, vol. $39, n^{\circ} 6$, p. 831-838, 2007.

Desceliers C., Soize C., Grimal Q., Haiat G., Naili S., “ A time domain method to solve transient elastic wave propagation in a multilayer medium with a hybrid spectral-finite element space approximation”, Journal of Wave Motion, vol. 45, n 4, p. 383-399, 2008.

Duchereau J., Soize C., " Transient dynamics in structures with nonhomogeneous uncertainties induced by complex joints", Mechanical Systems and Signal Processing, vol. 20, n 4 , p. 854-867, 2006.

Durand J.-F., Soize C., Gagliardini L., “ Structural-acoustic modeling of automotive vehicles in presence of uncertainties and experimental identification and validation", Journal of the Acoustical Society of America, vol. 124, n 3, p. 1513-1525, 2008.

Faverjon B., Ghanem R., "Stochastic inversion in acoustic scattering”, Journal of the Acoustical Society of America, vol. 119, n 6, p. 3577-3588, 2006.

Geman S., Geman D., " Stochastic relaxation, Gibbs distribution and the Bayesian distribution of images", IEEE Transactions on Pattern Analysis and Machine Intelligence, vol. Vol PAM I-6, n 6, p. 721-741, 1984.

Ghanem R., Kruger R. M., “ Numerical solution of spectral stochastic finite element systems”, Computer Methods in Applied Mechanics and Engineering, vol. 129, p. 289-303, 1996.

Ghanem R., Masri S., Pellissetti M., Wolfe R., “ Identification and prediction of stochastic dynamical systems in a polynomial chaos basis", Computer Methods in Applied Mechanics and Engineering, vol. 194, n 12-16, p. 1641-1654, 2005.

Ghanem R., Spanos P. D., Stochastic finite elements: a spectral approach, Springer-Verlag, New York, 1991.

Guilleminot J., Soize C., Kondo D., Benetruy C., “ Theoretical framework and experimental procedure for modelling volume fraction stochastic fluctuations in fiber reinforced composites", International Journal of Solid and Structures, vol. 45, n² 21, p. 5567-5583, 2008.

Hastings W. K., "Monte Carlo sampling methods using Markov chains and their applications", Biometrika, vol. 109, p. 57-97, 1970. 
Jaynes E. T., “ Information theory and statistical mechanics”, Physical Review, vol. 108, n² 2 , p. 171-190, 1957.

Kaipio J., Somersalo E., Statistical ans Computational Inverse Problems, Springer-Verlag, New York, 2005.

LeMaitre O. P., Najm H. N., Ghanem R., Knio O., “ Multi-resolution analysis of Wiener-type uncertainty propagation schemes”, Journal of Computational Physics, vol. 197, n², p. 502 531,2004

LeMaitre O. P., Najm H. N., Pebay P. P., Ghanem R., Knio O., “ Multi-resolution-analysis scheme for uncertainty quantification in chemical systems", SIAM Journal on Scientific Computing, vol. 29, $\mathrm{n}^{\circ}$ 2, p. 864-889, 2007.

Mace R., Worden W., Manson G., " Uncertainty in Structural Dynamics”, Special issue of the Journal of Sound and Vibration, vol. 288, n 3, p. 431-790, 2005.

MacKeown P. K., Stochastic Simulation in Physics, Springer-Verlag, Singapore, 1997.

Mehta M. L., Random Matrices, Revised and Enlarged Second Edition, Academic Press, New York, 1991.

Mignolet M. P., Soize C., " Nonparametric stochastic modeling of linear systems with prescribed variance of several natural frequencies", Probabilistic Engineering Mechanics, vol. 23, n 2-3, p. 267-278, 2008a.

Mignolet M. P., Soize C., “ Stochastic reduced order models for uncertain nonlinear dynamical systems", Computer Methods in Applied Mechanics and Engineering, vol. 197, n 45-48, p. 3951-3963, 2008b.

Muravyov A. A., Rizzi S. A., " Determination of nonlinear stiffness with application to random vibration of geometrically nonlinear structures", Computers and Structures, vol. 81, n 15 p. 1513-1523, 2003.

Nouy A., “ A generalized spectral decomposition technique to solve a class of linear stochastic partial differential equations", Computer Methods in Applied Mechanics and Engineering, vol. 196, n 45-48, p. 4521-4537, 2007.

Nouy A., Maitre O. P. L., " Generalized spectral decomposition for stochastic nonlinear problems”, Journal of Computational Physics, vol. 228, n 1, p. 202-235, 2009.

Ohayon R., Soize C., Structural Acoustics and Vibration, Academic Press, San Diego, London, 1998.

Pellissetti M., Capiez-Lernout E., Pradlwarter H., Soize C., Schueller G. I., “ Reliability analysis of a satellite structure with a parametric and a non-parametric probabilistic model", Computer Methods in Applied Mechanics and Engineering, vol. 198, $\mathrm{n}^{\circ}$ 2, p. 344-357, 2008.

Sampaio R., Soize C., “ On measures of non-linearity effects for uncertain dynamical systems - Application to a vibro-impact system", Journal of Sound and Vibration, vol. 303, n 3-5, p. 659-674, 2007.

Schueller G. I., " Computational methods in stochastic mechanics and reliability analysis", Special issue of Computer Methods in Applied Mechanics and Engineering, vol. 194, n ${ }^{\circ} 12$ 16, p. 1251-1795, 2005a.

Schueller G. I., " Uncertainties in structural mechanics and analysis-computational methods", Special issue of Computer and Structures, vol. 83, n 14, p. 1031-1150, 2005b.

Serfling R. J., Approximation Theorems of Mathematical Statistics, John Wiley \& Sons, 1980. 
Shannon C. E., “ A mathematical theory of communication”, Bell System Technology Journal, vol. 27, n 14, p. 379-423 \& 623-659, 1948.

Soize C., The Fokker-Planck Equation for Stochastic Dynamical Systems and its Explicit Steady State Solutions, World Scientific Publishing Co Pte Ltd, Singapore, 1994.

Soize C., “ A nonparametric model of random uncertainties on reduced matrix model in structural dynamics", Probabilistic Engineering Mechanics, vol. 15, n 3, p. 277-294, 2000.

Soize C., " Maximum entropy approach for modeling random uncertainties in transient elastodynamics", Journal of the Acoustical Society of America, vol. 109, n 5, p. 1979-1996, 2001.

Soize C., "Uncertain dynamical systems in the medium-frequency range", Journal of Engineering Mechanics, vol. 129, n 9, p. 1017-1027, 2003.

Soize C., "Random matrix theory for modeling uncertainties in computational mechanics", Computer Methods in Applied Mechanics and Engineering, vol. 194, n ${ }^{\circ} 12-16$, p. $1333-$ 1366, 2005.

Soize C., " Non Gaussian positive-definite matrix-valued random fields for elliptic stochastic partial differential operators", Computer Methods in Applied Mechanics and Engineering, vol. 195, n 1-3, p. 26-64, 2006.

Soize C., "Construction of probability distributions in high dimension using the maximum entropy principle. Applications to stochastic processes, random fields and random matrices", International Journal for Numerical Methods in Engineering, vol. 76, n 10, p. 1583-1611, $2008 \mathrm{a}$.

Soize C., " Tensor-valued random fields for meso-scale stochastic model of anisotropic elastic microstructure and probabilistic analysis of representative volume element size", Probabilistic Engineering Mechanics, vol. 23, n²-3, p. 307-323, $2008 \mathrm{~b}$.

Soize C., "Nonparametric probabilistic approach of uncertainties for elliptic boundary value problem", International Journal for Numerical Methods in Engineering, vol. 80, n 6-7, p. 673-688, 2009.

Soize C., "Generalized Probabilistic approach of uncertainties in computational dynamics using random matrices and polynomial chaos decompositions", International Journal for Numerical Methods in Engineering, vol. 81, n 8, p. 939-970, 2010.

Soize C., Capiez-Lernout E., Durand J.-F., Fernandez C., Gagliardini L., “ Probabilistic model identification of uncertainties in computational models for dynamical systems and experimental validation", Computer Methods in Applied Mechanics and Engineering, vol. 198, $\mathrm{n}^{\circ} 1$, p. 150-163, 2008.

Soize C., Ghanem R., “Physical systems with random uncertainties : Chaos representation with arbitrary probability measure", SIAM Journal On Scientific Computing, vol. 26, n², p. 395-410, 2004.

Soize C., Ghanem R., “ Reduced chaos decomposition with random coefficients of vectorvalued random variables and random fields", Computer Methods in Applied Mechanics and Engineering, vol. 198, n 21-26, p. 1926-1934, 2009.

Spall J. C., Introduction to Stochastic Search and Optimization, John Wiley, 2003.

Wiener N., " The Homogeneous Chaos", American Journal of Mathematics, vol. 60, $\mathrm{n}^{\circ} 1$, p. 897-936, 1938. 